\title{
Book \& New Media Reviews
}

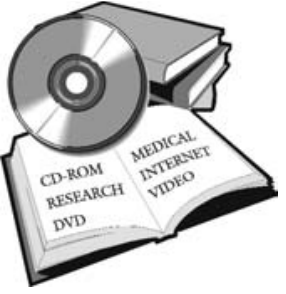

\section{CD-ROM Review}

Clinical Anesthesia for the PDA

Paul G. Barash, Bruce F. Cullen, Robert K. Stoelting. Lippincott Williams and Wilkins, 2002. $\$ 60.00$ US. ISBN 0-7817-3852-0

The Personal Data Assistant (PDA) has now entered into clinical practice in a very big way. PDAs can provide clinicians with a handheld tool to manage patient information, create logs of procedures performed, store clinical guidelines, and even perform clinical calculations. These miniature computers usually also have general tools such as word processors, spreadsheets, and personal scheduling software. One particularly popular clinical PDA application is ePocrates $\mathrm{Rx}$, a free drug reference program with over 2700 drug monographs and formulary information. This product, available at no cost at http://www.epocrates.com, can confirm drug dosages, identify generic preparations, evaluate potential interactions, and more. Information on other free medical packages for the PDA is available at http://www.freewarepalm.com/medical/medical.shtml.

However, until recently, there has been little of the way of PDA resources specifically for anesthesiologists. Now, with the publication of Clinical Anesthesia for the $P D A$ the situation has finally changed. Intended for both the working anesthesiologist and the resident in training, this product is based on the fourth edition of the Handbook of Clinical Anesthesia by Drs. Paul Barash, Bruce Cullen, and Robert Stoelting. Loaded with easily accessible protocols, tables and formulas, Clinical Anesthesia for the PDA covers all aspects of clinical anesthesia management in a succinct and convenient form. A comprehensive drug database even includes information on antibiotics and herbal medications. Also included are the Malignant Hyperthermia Association of the United States and American Heart Association resuscitation protocols, subacute bacterial endocarditis precaution information, information on difficult airway management, and automatic implantable cardioverter defibrillator information.

Purchasers of the CD-ROM get two versions of the software, one for the Palm platform and one for Pocket PC devices. PDA memory requirement in both cases are surprisingly modest: $\mathrm{l} \mathrm{MB}$ and $1.75 \mathrm{MB}$ respectively. If you prefer not to order the product from Lippincott Williams and Wilkins, you can download it instantly from Skyscape (http://www.skyscape.com).

The organization of the materials is divided into the following sections, each of which is in turn subdivided into numerous subsections:

I. Introduction to Anesthesia Practice

II. Basic Principles of Anesthesia Practice

III. Basic Principles of Pharmacology in Anesthesia Practice

IV. Preparing for Anesthesia

V. Management of Anesthesia

VI. Postanesthesia and Consultant Practice

VII. Appendixes

I had no difficulty installing the software on my Sony Clie PDA (a Palm-based system) and I found the software to be intuitive and very easy to use. The information was especially well formatted for display on small PDA screens. Finally, a search function makes it especially easy to find information quickly.

I recommend this product without reservation. Every anesthesiologist with a PDA should have it.

D. John Doyle MD PhD FRCPC

Cleveland, Ohio

\section{Respiratory Critical Care}

Craig Davidson, David Treacher (Eds.). Oxford

University Press, 2002. 307 pages. \$156.00 (CDN). ISBN 0-340-76289-6

This book was written by an international panel of well known experts, mainly from Europe, Australia and Canada. It is destined to respiratory physicians who work in critical care units. The initial chapters are an overview of pulmonary physiology, basics in mechanical ventilation basis and ventilation strategies. Monitoring and diagnostic methods are described subsequently. These chapters do not add any new concepts and simply review well known literature. 
The most interesting part of the book consists of chapters dealing with the clinical problems encountered in the intensive care unit. The chapters are an excellent summary of all the latest knowledge on the pathophysiology, diagnosis and management of these problems. Despite some duplication from chapter to chapter, the information will be useful to critical care physicians.

Overall this (almost pocket) book provides good clues to the bedside practitioner faced with common clinical respiratory problems in critical care medicine.

Stephan Langevin MD FRCPC

Québec, Québec

\section{NMS Clinical Manuals. Anesthesiology}

Randall S. Gliddden. Lippincott Williams \& Wilkins, 2003. 186 pages. $\$ 18.95$ (US). ISBN 0-7817-3738-9

A group of 14 clinical teachers from Boston have realized the need for this pocket-sized manual in undergraduate programs. Seventeen chapters describe historical background (chapter I), the clinical anesthesia encounter (part I, chapters 2-13) and Anesthesia specialty areas (part II, chapters 14-17).

In such a small textbook with such small print one would expect heavy reliance on algorithms, tables and point-form summaries. An unexpected paragraph-style format stimulates interest to "read on" and the student finds surprising detail and interesting information.

The attempt to follow Society for Education in Anesthesia guidelines is important as much thought and discussion at the ad hoc undergraduate Medical Student Committee has outlined clear and realistic objectives in the past.

Algorithms and tables are not abundant but make appropriate and succinct contributions to the text. Monitoring standards (chapter 6) are current and an extensive list of references reflect their complexity and importance.

Perhaps due to the sheer scope of the topic, part II, anesthesia specialty areas, is more difficult to cover. References are less specific and include large textbooks of material. Nevertheless, the basic information may further stimulate students' interest.

To facilitate improved readability the print and the manual itself (it is very small) could be larger and still remain pocket-sized.

Frances Barry MD

Montreal, Quebec 